\title{
The MHD coupling between coronal dynamics and photospheric motions
}

\author{
R. Grappin ${ }^{1}$, G. Aulanier ${ }^{2}$, and R. Pinto \\ 1 Observatoire de Paris, LUTH, CNRS, 92195 Meudon, France \\ e-mail: Roland.Grappin@obspm. fr \\ 2 Observatoire de Paris, LESIA, CNRS, 92195 Meudon, France \\ e-mail: guillaume.Aulanier@obspm.fr
}

Received 13 June 2008 / Accepted 20 August 2008

ABSTRACT

\begin{abstract}
Context. Whether it be the heating problem or the destabilization of coronal structures, use is often made of the so-called "linetying" boundary conditions, which amounts to imposing the photospheric velocity at the photosphere as a boundary condition for coronal dynamics. Directly coupling the low beta coronal evolution to prescribed photospheric motions of the magnetic footpoints allows strong magnetic energy accumulation in the corona. But this amounts to ignoring possible feedback from the coronal loops on photospheric motions, a neglect that is commonly justified by the strong density contrast between the photosphere and the corona. On the other hand, the energy injected into the corona comes from the photosphere, so in principle the coronal loop might act as a conduit communicating photospheric dynamics from one region to another.

Aims. Our objective is to test the degree of validity of this line-tying approximation by considering the role of the dense photosphere explicitly.

Methods. We consider here a 1.5D MHD model of a magnetic loop including a strongly stratified solar-like atmosphere and consider free (instead of prescribed/line-tied) boundary conditions applied deep in the photosphere, so as to quantify the coupling between the photosphere and corona as determined by stratification. We give an initial kick to one of the footpoints in the form of an upwardly propagating Alfvénic perturbation rising from the lower boundary, and then allow waves to freely escape the numerical domain from the boundaries, seated deep in the photosphere.

Results. We find that the response of the loop differs in many aspects from what is predicted by the line-tied condition. a) The magnetic energy density available in the corona is limited to a value equal to the kinetic energy density in the photospheric motion. b) The initial velocity shear between the opposite loop footpoints vanishes after a time proportional to the loop length. The shear between the coronal boundaries on opposite sides of the loop is quasi-uniform and is relaxed slowly by Alfvén waves propagating downwards through the high- $\beta$ photospheric layers. This process is insensitive to details of the thermal structure. c) Coronal loops are thus shown to exert a strong feedback on the photospheric dynamics, intermediate between friction and diffusion, instead of no reaction at all.
\end{abstract}

Key words. magnetohydrodynamics (MHD) - waves - Sun: magnetic fields - Sun: atmosphere - stars: atmospheres

\section{Introduction}

The line-tying condition applied at the corona-photosphere interface amounts to prescribing photospheric velocities, neglecting any feedback from the coronal dynamics on photospheric motions. These boundary conditions allow large magnetic energy accumulation in the corona, as field lines are slowly sheared and twisted by slow photospheric motions, and is helpful when dealing with the coronal heating issue (e.g. Rappazzo et al. 2007) or with the equilibrium of coronal structures (e.g. Velli \& Hood 1989; Aulanier et al. 2005), which asks for long time integrations that make the detailed modeling of the subcoronal region a numerically challenging task (e.g. Archontis \& Hood 2008; Martinez-Sykora et al. 2008).

The line-tying condition amounts to considering the photosphere as providing complete reflection of any coronal disturbance impinging from above. This is supported by early studies by Leroy (1980), Hollweg (1984), and more recently by Ofman (2002) and Gruszecki et al. (2007); however, these studies deal with finite frequency oscillations, not with slow photospheric movements.
To test the validity of the line-tied condition in the lowfrequency limit, we consider here a simple model problem. We consider a standard 1.5D MHD model of a coronal loop of length $L$ with uniform axial field $B_{0}$, including gravitational stratification, and examine the response of the loop when we give an initial kick to the left loop footpoint $u_{\perp}(x=0)=U_{0}$, in the form of an Alfvénic perturbation propagating from below the lower boundary seated deep in the photosphere.

We do not perturb the loop further, leaving any perturbation free to leave the numerical domain without reflection at the simulation boundaries, which are taken to be transparent using the standard technique of incoming and outgoing characteristics (see e.g. Grappin et al. 2000). Physical reflection inside the domain due to the stratification is of course taken into account. The boundary between photosphere and corona is well inside our numerical domain.

We then examine how the initial perturbation is transmitted or not to the right footpoint at $x=L$, and how much additional magnetic energy due to $b_{\perp}$ can be stored in the corona (at $x=L / 2$ ).

The kick in $u_{\perp}$ propagates first as an Alfvén wave along the loop. Assuming the WKB limit to be valid, a transverse 
magnetic field $b_{\perp}$ propagates as well, $b_{\perp} / B_{0}=-u_{\perp} / v_{\mathrm{A}}, v_{\mathrm{A}}$ being the Alfvén speed. The coronal amplitude of $b_{\perp}$ is weak compared to its photospheric value, since the approximate conservation of wave energy flux leads to a decrease with density $\rho$ as $b_{\perp} \sim \rho^{1 / 4}$, hence

$b_{\perp}^{c} / B_{0}=\left(U_{0} / v_{\mathrm{A}}^{0}\right)\left(\rho^{c} / \rho^{0}\right)^{1 / 4}$,

the index $c$ (resp. 0) denoting the coronal (resp. photospheric) value.

Further evolution depends on the boundary conditions. If line-tying is imposed at the opposite corona-photosphere boundary, $b_{\perp}$ grows uniformly along the loop linearly with time:

$b_{\perp} / B_{0}=U_{0} t / L$.

In the other limit, if boundary velocities are free to vary and if no reflection occurs, the velocity shear vanishes when the transverse momentum given at one footpoint reaches the other one, i.e., after one Alfvén traversal time $\tau_{\mathrm{A}}$, and the maximum value reached by $b_{\perp}$ is limited to the value reached at that moment in Eq. (2), namely:

$b_{\perp} / B_{0}=U_{0} /\left\langle v_{\mathrm{A}}\right\rangle$

where $\left\langle v_{\mathrm{A}}\right\rangle=L / \tau_{\mathrm{A}}$ is the average Alfvén speed along the loop. Given the much higher density in the photosheric sections of our loop, this average is dominated by the travel time in the phospheric layers.

In this paper, we will show that the asymptotic magnetic energy actually brought to the corona by photospheric shear is in between the two previous cases.

\section{Equations, physics, and method}

We consider a semi-circular loop (Fig. 1, left) of constant crosssection with uniform magnetic field $B_{0}=100 \mathrm{G}$. We neglect for simplicity $2 \mathrm{D}$ effects such as the curvature terms. Consequently, we consider the loop as a straight cylinder confined between two planes representing the footpoints (Fig. 1, right). We denote by $x$ the direction parallel to the initial field, and by $z$ the horizontal direction perpendicular to the plane of the loop. We consider no $y$-polarization. The velocity shear along the loop is thus represented by $u_{\perp}(x)=u_{z}(x)$, and the magnetic shear by $b_{\perp}(x)=b_{z}(x)$. The one-dimensional MHD equations read, together with the equation of state, as

$$
\begin{aligned}
& \partial_{t} \rho+\partial_{x}\left(\rho u_{x}\right)=0, \\
& \partial_{t} u_{x}+u_{x} \partial_{x} u_{x}+(1 / \rho) \partial_{x}\left(P+b_{z}^{2} / 2\right)=g(x)+v \partial_{x x} u_{x}, \\
& \partial_{t} u_{z}+u_{x} \partial_{x} u_{z}=B_{0} \partial_{x} b_{z}+v \partial_{x x} u_{z}, \\
& \partial_{t} b_{z}=-b_{z} \partial_{x} u_{x}-u_{x} \partial_{x} b_{z}+B_{0} \partial_{x} u_{z}, \\
& \partial_{t} T+u \partial_{x} T+(\gamma-1) T \partial_{x} u_{x}=\kappa \partial_{x x}\left(T-T_{0}\right), \text { and } \\
& P=2 n k T .
\end{aligned}
$$

The initial magnetic field is in the $x$-direction, but when subjected to a shear $u_{z}(x)$, it will turn, thus adding a $b_{z}$ component. Here $n\left(\rho=m_{p} n\right)$ is the density and $\gamma=1$. Due to $\nabla . B=0, b_{x}$ remains constant and equal to $B_{0}$. A very small $b_{y}$ is present from the start, to prevent the boundary equations to become singular. The viscosity is $v=U_{0} L / 1280$ and conductivity $\kappa=U_{0} L / 128$. Both are there for numerical stabilization purposes and play no other role in the results, as seen in our simulations when changing their values by a factor of 10 . Gravity projected on the semicircular loop is

$g(x)=-g_{0} \cos (\pi x / L)$

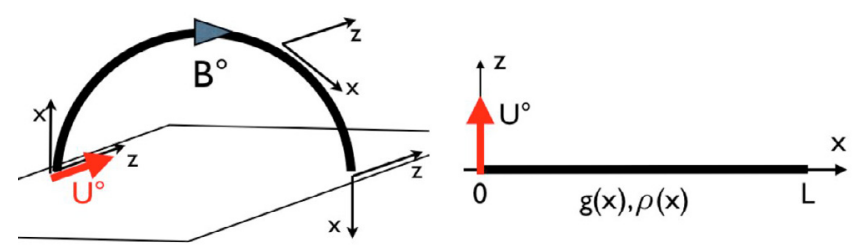

Fig. 1. Sketch of the model. A coronal loop is considered as a straight magnetic field line with density and gravitational acceleration varying along the axis of the line. A transverse kick is given to the left footpoint at $x=0$, during $0 \leq t \leq \tau_{0}$, see Eq. (14).

where $g_{0}$ is surface gravity. In Eq. (8), $T_{0}(x)$ is the temperature profile of the atmosphere:

$T_{0}(x)=T_{a}+\left(T_{b}-T_{a}\right) \tanh \left(\left(x-x_{0}\right) / \delta x\right)$

where $T_{a}=6000 \mathrm{~K}, T_{b}=1 \mathrm{MK}, x_{0}=7 \mathrm{Mm}$, and $\delta x=0.7 \mathrm{Mm}$ are the position and width of the transition region. We found that the thermal structure may be changed (for instance taking $x_{0}=2 \mathrm{Mm}$ and $\delta x=0.2 \mathrm{Mm}$ ) without any effect on the results. The same is true when changing $\gamma$ to $5 / 3$ instead of 1 . The initial density profile $\rho(x)$ is computed by integrating the hydrostatic equilibrium.

We use a Runge-Kutta temporal scheme of order 3, a compact finite difference scheme of order 6 in the spatial domain (Lele 1992), and a uniform grid of $N=512$ points. Time step is automatically adapted using a priori estimations of the characteristic times.

The boundary conditions are imposed on the incoming characteristics of the equations (e.g. Grappin et al. 2000): the numerical domain boundaries, situated deep in the photosphere, are transparent to outgoing waves. In practice, time variation of each field at the boundary is split into two contributions, that of incoming waves and that of outgoing waves (characteristics). The outgoing part, communicating variations due to modes propagating from the inside out, are calculated from the values of the fields inside the domain, while the incoming characteristics are assigned as a given function of time.

In the present case, ingoing perturbations are defined at $x=0$ to generate a finite transverse velocity $u_{z}=U_{0}$ at the left footpoint, no flow along the axis, $u_{x}=0$, while no incoming perturbations are introduced at the opposite boundary.

We thus integrate at the left boundary the following equations for $u_{z}$ and $b_{z}$ (the other components are of less concern here):

$\partial_{t} u_{z}=\frac{\mathrm{d} A}{\mathrm{~d} t}-\frac{u_{x}-v_{\mathrm{A}}}{2}\left(\partial_{x} u_{z}+\frac{\partial_{x} b_{z}}{\sqrt{\rho}}\right)$
$\frac{\partial_{t} b_{z}}{\sqrt{\rho}}=-\frac{\mathrm{d} A}{\mathrm{~d} t}-\frac{u_{x}-v_{\mathrm{A}}}{2}\left(\partial_{x} u_{z}+\frac{\partial_{x} b_{z}}{\sqrt{\rho}}\right)$

where $A(t)$ is a ramp reaching $U_{0}=-100 \mathrm{~m} / \mathrm{s}$ in a time $\tau_{0}=$ $100 \mathrm{~s}:$

$A(t)=U_{0}\left(1-\exp \left(-\left(t / \tau_{0}\right)^{4}\right)\right)$.

At the right footpoint $x=L$, the equations for $u_{z}$ and $b_{z}$ are the same as at the left footpoint, but with $A(t)$ replaced by zero.

At short times, Eqs. (12), (13) imply that, at $x=0, u_{z}=A(t)$ and (neglecting density fluctuations at the photosphere) $b_{z} / \sqrt{\rho}=$ $-A(t)$, so that the left footpoint quickly approaches the speed $U_{0}=-100 \mathrm{~m} / \mathrm{s}$ and $b_{z}=14.5 \mathrm{G}$ (i.e., $b_{z} / \sqrt{\rho}=100 \mathrm{~m} / \mathrm{s}$ ). This will remain so as long as there is no loop feedback in the form of a reflected wave with $u_{z}+b_{z} / \sqrt{\rho} \neq 0$. Recall that with 

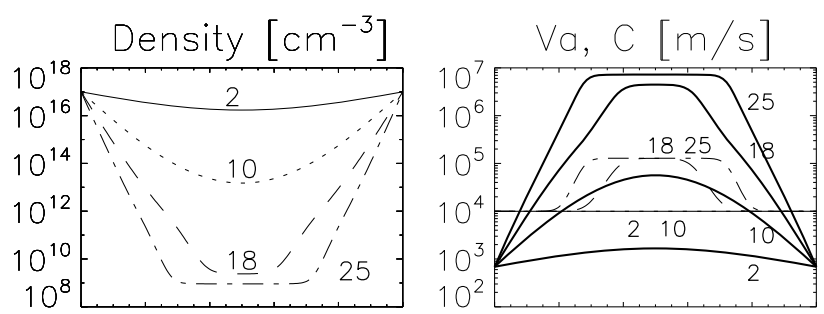

$\begin{array}{llllllll}0.0 & 0.2 & 0.4 & 0.6 & 0.8 & 1.0\end{array}$ $\times / L$ $\begin{array}{llllllll}0.0 & 0.2 & 0.4 & 0.6 & 0.8 & 1.0\end{array}$ $\times / L$

Fig. 2. Density (left), Alfvén (right, thick lines), and sound speed profiles (right, thin lines) vs. abscissa $x$ along loop, for loop lengths 2, 10, 18 , and $25 \mathrm{Mm}$ (resp. solid, dotted, dashed, and dotted-dashed, except Alfvén speed having all lines solid).
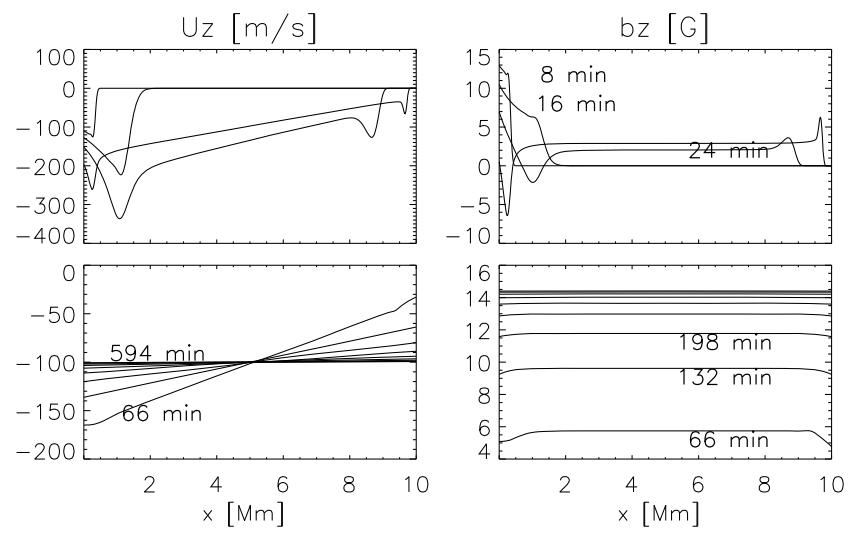

Fig. 3. Response of the $10 \mathrm{Mm}$ loop to a $-100 \mathrm{~m} / \mathrm{s}$ drift of the left footpoint. Top: first transit time; Bottom: further relaxation. $u_{z}$ (left) and $b_{z}$ profiles (right). Abscissa, coordinate $x$ along loop.

the line-tied boundary condition, $b_{z}$ would grow continuously, precisely because the direct wave is completely reflected by the right footpoint.

In summary, in our 1.5D loop model, there are only two sources of motions for the loop footpoints: either the subphotospheric part of the loop (outside the numerical domain, represented by the boundary conditions) or the atmospheric part of the loop itself within the numerical domain. Hence, after the initial kick has been given to the left footpoint (i.e., when $\mathrm{d} A / \mathrm{d} t$ has become negligible in Eqs. (12), (13), the only momentum given to the footpoints will come from the atmospheric part of the loop, with no perturbation and no constraint coming from the photospheric boundary.

We considered several loop lengths: $L=2,10,18$, and $25 \mathrm{Mm}$; the corresponding Alfvén and sound speed profiles are shown in Fig. 2.

\section{Results}

We show in Fig. 3 the evolution of $u_{z}$ and $b_{z}$ profiles in a $10 \mathrm{Mm}$ loop. This loop has no thermal transition region, as its middle part actually does not reach the corona, but note that when considering a lower transition region so as to include it within the loop, we obtain quasi-identical results. The top shows the propagation to the right footpoint, which takes about $40 \mathrm{~min}$. After some transient oscillations, $u_{z}$ and $b_{z}$ relax everywhere to their initial "kick" values (resp. $-100 \mathrm{~m} / \mathrm{s}$ and $14.5 \mathrm{G}$ ). This takes about $5 \mathrm{~h}$. A shorter loop ( $2 \mathrm{Mm}$ ) leads to similar behavior, but with a shorter relaxation time of about $1 \mathrm{~h}$.
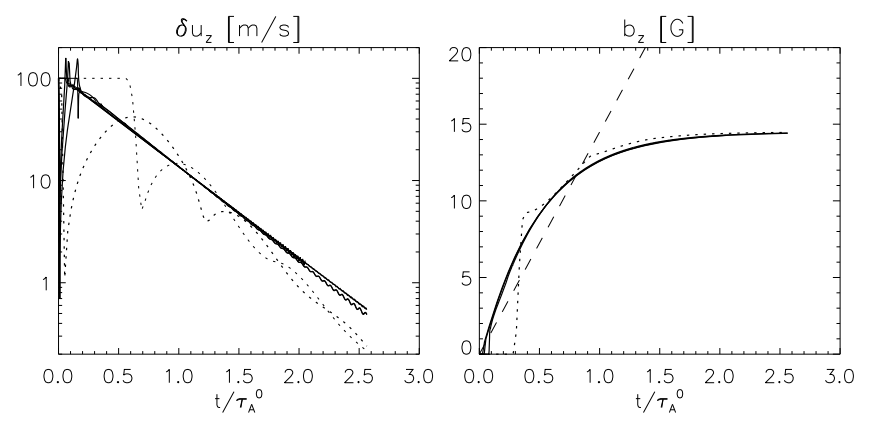

Fig. 4. Relaxation of velocity shear and saturation of magnetic shear: 2, 10, 18 and $25 \mathrm{Mm}$ loops (dotted lines for $2 \mathrm{Mm}$, solid lines for all others). Abscissa: time normalized by $\tau_{\mathrm{A}}^{0}$ (see text). Left: $\delta u_{z}=\left|u_{z}-U^{0}\right|$ at left and right footpoints $\left(U^{0}=-100 \mathrm{~m} / \mathrm{s}\right)$. Right: $b_{z}$ at apex; dashed line: line-tied result (Eq. (2)).

Increasing the loop length $L$ (including a transition region and a coronal region in the middle part of the loop) shows that the relaxation always occurs, the time increasing linearly with loop length. This is summarized in Fig. 4, in which one shows the time variation of the deviation from the asymptotic speed $\delta u_{z}=\left|u_{z}-U^{0}\right|$ at both footpoints for loop lengths 2, 10, 18, and $25 \mathrm{Mm}$, as well as the magnetic field $b_{z}$ at the apex. The time is normalized by $\tau_{\mathrm{A}}^{0}$, which is the traversal time of a loop length $\mathrm{L}$ for a fictitious Alfvén wave traveling everywhere with the photospheric Alfvén velocity $v_{\mathrm{A}}^{0}$ :

$\tau_{\mathrm{A}}^{0}=L / v_{\mathrm{A}}^{0}$.

A fit of the curves for the three longest loops gives

$\delta u_{z}=C \exp \left(-2.1 t / \tau_{\mathrm{A}}^{0}\right)$

The asymptotic $b_{z}$ is independent on the position $x$ (that is, the loop is straight), and is $14.5 \mathrm{G}$, i.e., additional magnetic energy is all along the loop equal to the photospheric kinetic energy:

$b_{z} / B_{0}=U_{0} / v_{\mathrm{A}}^{0}$.

This is greater than the purely "transparent" expression (Eq. (3)), and is actually the result reached by the line-tied expression (Eq. (2)) after one "photospheric" Alfvén time (Fig. 4, right). We note that the relaxation times are the same for $b_{z}$ and $u_{z}$. The figure shows all curves nicely superposed, except the $2 \mathrm{Mm}$ loop, which relaxes more rapidly; however, a global fit including the $2 \mathrm{Mm}$ loop can be obtained by redefining the time scale as $\tau_{\mathrm{A}}^{0}=\left(L-L^{0}\right) / v_{\mathrm{A}}^{0}$, with $L^{0}=0.3 \mathrm{Mm}$.

\section{Discussion}

The exponential relaxation (16) just found can be explained by considering the global velocity shear as trapped in the high- $\beta$ region and leaking progressively. The relation (16) is finally recovered by assuming the logarithm of the reflexion coefficient to be given by the ratio of photospheric over coronal Alfvén speeds, which is compatible with classical ideas on reflection (Ofman 2002). Another explanation not relying on wave trapping is proposed below.

Figure 5 gives a view of the whole process. It shows in a space-time $(x, t)$ diagram the time derivative $\partial u_{z} / \partial t$ for the $10 \mathrm{Mm}$ loop, during the first two transit times (left panel) and during a later period of four Alfvén times (right panel). The left image shows how the transverse velocity signal propagates upward, with the Alfvén characteristics crossing the loop apex 

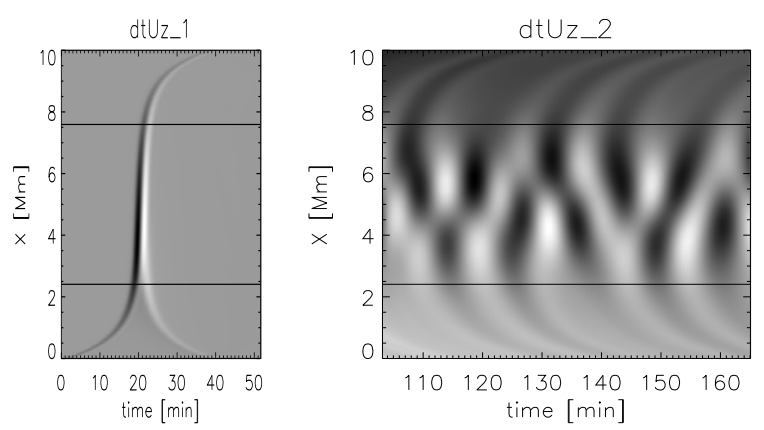

Fig. 5. Space-time diagrams for $\partial u_{z} / \partial t, 10 \mathrm{Mm}$ loop. Left: first Alfvén time showing propagation and reflexion of the first Alfvén signal from the left to the right footpoints. Right: later phase showing the slow relaxation of the large scale shear by Alfvén waves propagating downwards from the $\beta=1$ region (solid lines).

rapidly, and finally reaching the right footpoint at the same time as a strong reflected component reaches the left footpoint. This reveals the large wave reflection by the upper part of the loop, which then accelerates the left footpoint, and is also responsible for the right footpoint getting only a small part of the momentum at that time.

We now concentrate on the relaxation phase. The apex has $u_{z}=-100 \mathrm{~m} / \mathrm{s}$, but there is global shear: the left footpoint proceeds faster, and the right footpoint is slower (Fig. 3, bottom left). The transverse magnetic field $b_{z}$ shows a plateau: the low$\beta$ region has a higher $b_{z}$ than the dense high- $\beta$ region, that is, there is a jump in the magnetic field at the $v_{\mathrm{A}}=c$ boundary, which is decreasing with time (Fig. 3, bottom right, $t=66 \mathrm{~min}$ ).

The $\partial u_{z} / \partial t$ contours shown in the right panel of Fig. 5 show distinct wave patterns. The low- $\beta$ region shows standing slow waves. (Since the mean field is now no longer parallel to the wave vector, the pure Alfvén polarization is absent during the relaxation phase.) These slow waves are quasi-acoustic waves, with velocity polarization quasi-parallel to the total field: $u_{z} / u_{x} \sim b_{z} / B_{0}$, the fluctuations of $b_{z}$ being very small. These waves are trapped within the low- $\beta$ region (the leakage time is much longer than our relaxation time) and are the remnant of the initial Alfvén perturbation and generated by mode coupling at that time. They are coupled with periodic quasi-Alfvén pulses propagating in the high- $\beta$ region, i.e., with mainly transverse fluctuations $u_{z}$ and $b_{z}$.

Let us focus on the $v_{\mathrm{A}}=c(\beta \simeq 1)$ boundary. The jump in $b_{z}$ there triggers, due to the magnetic field tension, a transverse (quasi-Alfvén) wave that tends to suppress this $b_{z}$ jump. Let us denote by $\delta t_{\star}$ the time during which the wave travels from the plateau boundary down to the surface. Since the wave suppresses the $b_{z}$ jump $\delta b_{z}$, it also carries a velocity jump $\delta u_{z}$, which has opposite signs at the left and right footpoints (as it should for Alfvén modes propagating in opposite directions), and a magnitude, when arriving at the solar surface, equal to $\delta b_{z} / \sqrt{\rho^{0}}$, where $\rho^{0}$ is the photospheric density. This decreases the total velocity shear between footpoints $\Delta u_{z}$ by the amount $\delta \Delta u_{z}=2 \delta b_{z} / \sqrt{\rho^{0}}$.

Now, during the same time $\delta t_{\star}$, the magnetic field jump at the $v_{a}=c$ boundary is actually regenerated. Indeed, since the velocity shear between footpoints is decreased but not suppressed, the transverse magnetic component increases by the amount $\delta b_{z}=\Delta u_{z} B^{0} \delta t_{\star} / L$. This double process of a slow increase of the mean $b_{z}$ in the low- $\beta$ region and the permanent emission of progressive Alfvén wave in the low- $\beta$ region is visible in Fig. 6, which shows a series of profiles of $u_{z}$ and $b_{z}$ during the end of the relaxation process for the $10 \mathrm{Mm}$ loop.
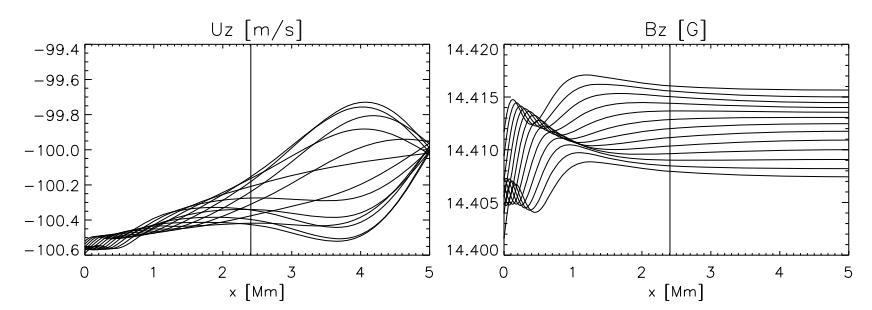

Fig. 6. $u_{z}$ (left) and $b_{z}$ (right) profiles in the left half of the $10 \mathrm{Mm}$ loop (vertical lines: $\beta=1$ layer). Alfvén waves are visible in the $\beta>1$ region. The $\beta<1$ region shows trapped acoustic $u_{z}$ oscillations and a steady $b_{z}$ increase due to the residual mean velocity shear.

Replacing the previous expression in the one for $\Delta u_{z}$, one has finally: $\mathrm{d} \Delta u_{z} / \mathrm{d} t \approx \delta \Delta u_{z} / \delta t_{\star}=-2\left(v_{\mathrm{A}}^{0} / L\right) \Delta u_{z}$, which leads to the same time scale as in Eq. (16).

The exchange of momentum between loop footpoints amounts to an anomalous diffusion, modifying the photospheric dynamics. In the photospheric $(y, z)$ plane, the diffusion term may be written as $\partial u_{i} / \partial t \sim-v_{\mathrm{A}}^{0}\left|u_{i}(X+L)-u_{i}(X)\right| / L \sim-v_{\mathrm{A}}^{0}\left|\nabla u_{i}\right|$, (see Eq. (16)) where $i=y, z$ and $X=(y, z)$; the $|\nabla|$ operator is intermediate between friction and Laplacian. Such a modeling of the effect of the corona on photospheric motions would be of interest compared to a complete simulation including photosphere and corona, or compared to the usual line-tied hypothesis, which completely ignores any feedback of the loops on the photospheric motions. It offers a viewpoint complementary to that of Erdélyi (2006). Further study, including in particular 2D effects, is desirable, in which case the role of the transition region might be more pronounced (see DelZanna et al. 2005).

We conclude that these results change the status of the linetied condition for slowly varying photospheric motions because the latter condition neglects the momentum exchanges between footpoints that are important even at short times. Our result that the coronal free magnetic energy equals the kinetic energy of the photospheric motions (Eq. (17)) puts no new strong limit on coronal heating. However, for the formation (and further destabilization) of highly-stressed coronal fields, such as prominences and sigmoids, our results imply that the largest part of the magnetic shear cannot be induced by simple horizontal photospheric motions. This conclusion is strictly valid within the low-frequency limit, for loops with constant cross-section, and neglecting transverse structuring. Work relaxing these assumptions is in progress to continue exploring how photospheric and coronal dynamics couple.

Acknowledgements. Financial support by the European Commission through the SOLAIRE Network (MTRN-CT-2006-035484) is gratefully acknowledged.

\section{References}

Aulanier, G., Démoulin, P., \& Grappin, R. 2005, A\&A, 430, 1067 Archontis, V., \& Hood, A. W. 2008, ApJ, 674, L113

Del Zanna, L., Shaekens, E., \& Velli, M. 2005, A\&A, 431, 1095 Erdélyi, R. 2006, Phil. Trans. R. Soc. A, 364, 351

Grappin, R., Léorat, J., \& Buttighoffer, A. 2000, A\&A, 362, 342

Gruszecki, M., Murawski, K., Solanki, S. K., \& Ofman, L. 2007, A\&A, 469, 1117

Hollweg, J. V. 1984, ApJ, 277, 392

Lele, J. 1992, Comput. Phys., 103, 16

Leroy, B. 1980, A\&A, 91, 136

Martinez-Sykora, J., Hansteen, V., \& Carlsson, M. 2008, ApJ, 679, 871

Ofman, L. 2002, ApJ, 568, L135

Rappazzo, A. F., Velli, M., Einaudi, G., \& Dahlburg, R. B. 2007, ApJ, 657, L47 Velli, M., \& Hood, A. W. 1989, Sol. Phys., 119, 107 